\title{
In Every Challenge There Lies Opportunity: Rise of the Phoenix
}

\author{
Richard Jones
}

$\mathrm{T}$

he title of this article clearly reflects the view of an optimist.

For how can something positive come out of every challenge? In basic terms, the subtitle refers to the phoenix, a mythical bird, rising from the ashes of its own funeral pyre to live again. In the context of modern life, business journals have for years reported case examples in which people have found opportunity where none was apparent to the masses. They forged ahead when all appeared lost. In the current economic recession, political and economic leaders are now suggesting that we have reached the bottom of the downturn, but in fact we are only beginning to see the very challenging impacts on hospital care services in Canada.

As just one example of these impacts, hospitals are being radically restructured into broad regional systems, even single province-wide organizations, in an effort to manage the cost of care while ensuring that as many public dollars as possible are spent on the delivery of care. Because of these changes, hospital leaders are being asked to accept even greater responsibilities; in response, some of these leaders are changing career paths, even moving out of care delivery altogether. Nonetheless, from this "bed of ashes", pharmacists and other health care providers are rising to the challenge of finding new ways to deliver care, including the creation of primary care clinics and specialty clinics, moving closer to the patient on every front. The shift to provision of patient care in more ambulatory settings, even within the hospital, is seeing a growing number of pharmacists ply their craft alongside physicians and nurses as essential members of the care team. Hospital leaders are finding ways to ensure that the appropriate infrastructure is present to allow these developments to occur, but the need for leaders and their skills as we have known them is changing as well.

Pharmacists are gradually becoming independent practitioners and thereby leaders of their own practices in a growing diversity of settings. Traditional leaders have often been difficult to find, but they will soon be replaced by people with new skills, for example, running a logistic, production, or distribution system or serving as a clinical practitioner-leader, akin to the leaders among our physician and nursing colleagues. Pharmacist specialties and their corresponding training and

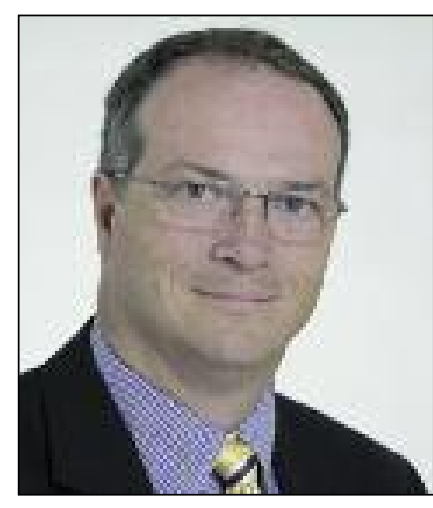
credentialling will form the basis of practice leadership for this new category of leaders. Registered pharmacy technicians will serve as the operational leaders of the future, fully freeing pharmacists to be practitioners.

The advent of new clinical pharmacist leaders will represent a "rise from the ashes" as their partnerships with physicians and nurses produce a new and comprehensive model of conjoint care. And just imagine: all of this will be achieved during a significant downturn in the economy and the resultant restructuring in Canadian health care. Many see these opportunities today and are working quietly to ensure that the rise from the ashes is strong, substantial, and enduring. Others do not yet see the opportunities. To fully realize the possibilities, we need to find the early leaders among our pharmacist, physician, and nursing peers and work with them as a team to deliver well-structured, efficient, and high-value clinical services to the patients, for whom we all care so deeply.

Richard Jones, RPh, BSC, BSP, is Past President and Internal Liaison for CSHP. 